\title{
Do Children of Long-Lived Parents Age More Successfully?
}

\author{
Henrik Frederiksen, ${ }^{1}$ Matt McGue, ${ }^{2}$ Bernard Jeune, ${ }^{1}$ David Gaist, ${ }^{1}$ Hanne Nybo, ${ }^{1}$ \\ Axel Skytthe, ${ }^{3}$ James W. Vaupel, ${ }^{4}$ and Kaare Christensen ${ }^{1,3}$
}

Background. Long-lived individuals are rare and may be selected in part for the genetic factors that promote successful aging. The children of long-lived parents may therefore age more successfully than the children of short-lived parents.

Methods. We used three major cross-sectional populationbased surveys to study the association of parental longevity with successful aging in offspring. The measures of aging were hand-grip strength, cognitive performance (Mini Mental State Examination and a cognitive composite score), self-reported diseases, and self-rated health.

Results. For every additional 10 years the parents lived, their children's grip strength increased by $0.32 \mathrm{~kg}(95 \% \mathrm{CI}=$ 0.00-0.63), Mini Mental State Examination score by 0.20 points $(95 \% \mathrm{CI}=0.03-0.37)$, and cognitive composite score by 0.24 points $(95 \% \mathrm{CI}=0.07-0.40)$. A 10 -year increment of parental life was associated with a reduction by approximately 0.20 in the adjusted odds ratio for their children having each of the following conditions: diabetes; hypertension; ischemic heart disease; heart failure; stroke; or fair, poor, or very poor self-rated health. Almost all the effects were seen solely in the cohort of $70+$-year-olds, but not among middle-aged or nonagenarian subjects.

Conclusions. Parental life span is positively associated with the children's physical and cognitive functioning and avoidance of some of the common chronic diseases. However, the effects are small and are seen among offspring who are elderly, but not among the middle-aged or the oldest old. (Epidemiology 2002;13:334-339)

Key words: aging, twins, oldest-old, parents, longevity, hand strength, cognition.

\section{LIPPIN}

$\mathrm{M}$ aintenance of high physical and cognitive functioning and avoidance of disease and disability are central to "successful" aging. Ever since Rowe and Kahn ${ }^{1}$ introduced the concept of successful aging, a number of mainly environmental predictors (eg, education, income, social network, physical activity, and smoking status) have been shown to influence the components of successful aging. ${ }^{2,3}$ In recent years, researchers have also begun to investigate the contribution of genetic factors to successful aging. ${ }^{4-6}$ Studies of elderly twins suggest that genetic factors account for a substantial proportion of the variance in

From the ${ }^{1}$ Institute of Public Health, Epidemiology, and ${ }^{3}$ Danish Center for Demographic Research, University of Southern Denmark, Odense; ${ }^{2}$ Department of Psychology, University of Minnesota, Minneapolis, MN; and ${ }^{4}$ Max Planck Institute for Demographic Research, Rostock, Germany.

Address correspondence to: Henrik Frederiksen, Institute of Public Health, Epidemiology, University of Southern Denmark, Sdr. Boulevard 23A, DK-5000 Odense C, Denmark; hfrederiksen@health.sdu.dk

Supported by U.S. National Institute on Aging Research Grant NIA-PO1AG08761, Sygekassernes Helsefond Grant 11/2 12-97, and the Danish National Research Foundation.

Submitted 3 July 2001; final version accepted 9 January 2002.

Copyright @ 2002 by Lippincott Williams \& Wilkins, Inc. physical $^{7}$ and cognitive ${ }^{8,9}$ functioning, and a moderate proportion of variance in life span. ${ }^{10}$ An important genetic component to some of the major diseases of aging, such as cardiovascular diseases, ${ }^{11}$ diabetes and impaired glucose tolerance, ${ }^{12}$ and some cancers (breast, prostate, and colorectal), ${ }^{13}$ has also been found. Long-lived individuals are rare and may be selected in part for the genetic factors that promote successful aging. The children of long-lived parents may therefore age more successfully than the children of short-lived parents. Whatever the mechanism, not much is known about the interplay between parental longevity and the aging of offspring. Here we use three major Danish nationwide surveys of middle-aged, elderly, and oldest-old people to study the association of parental life span with the respondents' physical and cognitive functioning, as well as specific illnesses.

\section{Methods}

\section{Sample}

The sample comprised participants in three nationwide population-based surveys: the Study of MiddleAged Danish Twins (MADT), ${ }^{14}$ the Longitudinal Study 
of Aging Danish Twins-3rd wave (LSADT), ${ }^{15}$ and the Danish 1905 Cohort Survey (1905), ${ }^{16}$ which have all been described in detail previously. In brief, participants in the MADT and LSADT studies were identified in the Danish Twin Register, ${ }^{17}$ and participants in the 1905 survey were identified in the Danish Civil Registration System, both nationwide, population-based registers that are continuously updated. The eligible participants in the MADT study represented a random sample of 120 twin pairs from each of the 22 birth cohorts in 19311952. The eligible participants in the LSADT were Danish twins age 70 years and older in 1999, and those in the 1905 study were all Danes born in 1905. All surveys were conducted in periods of 3-6 months in 1998-1999. The participation rates were $83.1 \%$ in the MADT $(\mathrm{N}=4,314), 69.9 \%$ in the LSADT $(\mathrm{N}=$ $2,709)$, and $62.8 \%$ in the 1905 cohort study $(\mathrm{N}=$ 2,262 ). All three studies used a highly comparable structured interview, which was home-based and included physical and cognitive testing performed by lay interviewers from the Danish National Institute of Social Research. All interviewers had substantial experience interviewing the elderly and furthermore completed a detailed training program by a physician and were closely monitored during the interview periods.

All studies were approved of by the Danish scientific ethical committees, and all participants provided informed consent.

\section{Parental Age at Death}

Information on parental age at death was obtained during the interview in the LSADT and 1905 studies and through a mailed questionnaire (1996-1998) before the MADT study. The twins within the intact pairs (both twins in a pair participating) reported their father's age at death with a correlation of 0.99 (95\% confidence interval $[\mathrm{CI}]=0.99-0.99)$ in the MADT study and $0.96(95 \% \mathrm{CI}=0.95-0.96)$ in the LSADT study, and their mother's age at death with a correlation of $0.98(95 \% \mathrm{CI}=0.98-0.99)$ in the MADT and 0.98 $(95 \%$ CI $=0.97-0.98)$ in the LSADT study. In the MADT and LSADT studies both twins in a pair were excluded if they differed by 5 or more years in their report of either their father's or mother's age at death ( $\mathrm{N}=244$ individuals). The average of the fathers' and mothers' ages at death, as well as fathers' and mothers' ages at death separately, was used in the analyses.

\section{Physical Functioning}

Hand-grip strength, which has been shown to reflect overall muscle strength, ${ }^{18}$ was measured with a hand dynamometer (Smedley's dynamometer TTM, Tokyo). We identified the maximum value of three measures with each hand in the LSADT and MADT studies, and of three measures with the preferred hand in the 1905 study. Participants with fewer than three attempts, or with a difference of $20 \mathrm{~kg}$ or more between two measures, were excluded from this analysis $(\mathrm{N}=268)$.

\section{Cognitive Functioning}

Integrated in the interview was a series of cognitive tests including the Mini Mental State Examination (MMSE) (in the LSADT and the 1905 studies only), a test of fluency (number of animals named in 1 minute), forward and backward digit span, and a modified 12 word learning test. The scores of the four latter tests were standardized and summed to form a cognitive composite score that was used in the analysis.

\section{Health}

In all surveys the participants were asked whether a physician had ever told them that they suffered from various diseases. Participants who answered "Yes, previously" or "Yes, currently" were subsequently asked to confirm that this was a diagnosis made by a doctor, before the answer was accepted as valid.

\section{Data Analysis}

We divided participants into five groups by average parental age at death $(<54,55-64,65-74,75-84$, and $85+)$ and compared these groups with regard to physical and cognitive functioning as well as a number of selfreported diseases. Several potential confounding characteristics (body mass index, smoking habits, alcohol consumption, and education) were confirmed to be unrelated to parental life span. Both parents were deceased for about half of the participants in the MADT study. In the LSADT study only 15 participants had one parent still alive, and in the 1905 cohort study none of the participants had living parents. All measures were age-and sex-adjusted by subtracting age (in 5-year intervals) and sex-specific means from the actual scores. ${ }^{19}$ The residuals are presented in the figures. In all subsequent analyses, participants with parents who on average died before age 54 were excluded $(\mathrm{N}=243)$ as a result of the large proportion of deaths not related to the biology of aging (eg, accidents and suicides) before this age. After this, the effect of increasing the mean parental age at death by 10 years was assessed by entering the categorized value as a continuous measure in a multivariate linear regression model for continuous outcomes (grip strength and cognitive tests) or in a logistic regression model for dichotomous outcomes (self-reported diseases), controlling for the age and sex of the participants. A model with parental age at death as a continuous variable and a model with parental age at death in 10 -year groups gave virtually the same results and comparable Bayesian information criteria. To account for the nonindependence of the observations on twins, twins from pairs in which both participated were ana- 
TABLE 1. Basic Characteristics of 9,285 Participants in Three Large Danish Population-Based Studies

\begin{tabular}{|c|c|c|c|c|c|c|}
\hline & \multicolumn{2}{|c|}{ MADT } & \multicolumn{2}{|c|}{ LSADT } & \multicolumn{2}{|c|}{1905} \\
\hline & Mean $\pm \mathrm{SD}$ & $\mathrm{N}$ & Mean \pm SD & $\mathrm{N}$ & Mean \pm SD & $\mathrm{N}$ \\
\hline $\begin{array}{l}\text { Age of participants } \\
\% \text { females } \\
\text { Father's age at death* } \\
\text { Mother's age at death* } \\
\text { Mean parental age at death*† } \\
\text { Grip strength } * \\
\text { MMSE score } \\
\text { Cognitive composite score }\end{array}$ & $\begin{array}{l}56.9 \pm 6.3 \\
49.0 \\
72.5 \pm 12.0 \\
73.6 \pm 13.3 \\
73.9 \pm 9.4 \\
38.1 \pm 12.4 \\
=- \\
4.6 \pm 3.5\end{array}$ & $\begin{array}{l}4,314 \\
2,116 \\
3,231 \\
2,466 \\
2,136 \\
4,148 \\
4,309\end{array}$ & $\begin{array}{l}78.3 \pm 6.0 \\
57.8 \\
74.4 \pm 12.8 \\
76.0 \pm 15.2 \\
75.3 \pm 10.1 \\
26.6 \pm 9.6 \\
25.8 \pm 5.4 \\
1.5 \pm 3.5\end{array}$ & $\begin{array}{l}2,709 \\
1,565 \\
2,403 \\
2,463 \\
2,281 \\
2,288 \\
2,565 \\
2,481\end{array}$ & $\begin{array}{l}93 \pm 0 \\
74.2 \\
74.5 \pm 14.1 \\
73.8 \pm 16.1 \\
74.1 \pm 10.8 \\
16.0 \pm 6.7 \\
21.4 \pm 6.0 \\
0.0 \pm 3.6\end{array}$ & $\begin{array}{l}2,262 \\
1,678 \\
1,877 \\
1,981 \\
1,835 \\
1,485 \\
1,798 \\
1,784\end{array}$ \\
\hline
\end{tabular}

MADT $=$ Study of Middle-Aged Danish Twins; LSADT = Longitudinal Study of Aging Danish Twins—3rd wave; $1905=$ Danish 1905 Cohort Study; MMSE = Mini Mental State Examination (in LSADT and 1905 studies only).

* Individuals in a twin pair are excluded if they reported the father's $(\mathrm{n}=152)$, mother's $(\mathrm{n}=140)$, or both parents' $(\mathrm{n}=244)$ age at death in the MADT and LSADT studies with 5 or more years' difference.

$\dagger$ For participants with both parents dead, average of fathers and mothers age at death.

\$ Maximum of at least three examinations with less than $20-\mathrm{kg}$ difference between two measures.

$\S$ A composite measure of test for fluency, forward and backward digit span, and a modified 12-word learning test.

lyzed as clusters of two in the multivariate models. Thirteen twins born in 1905 participated in both the 1905 and the LSADT survey. The data from both surveys of these participants were used in the analyses because they were so few and sampled with an interval of 6 months.

\section{Results}

Table 1 shows the basic characteristics of the participants in the three surveys. Table 2 shows the number of participants across categories of parental longevity. At least one parent was still alive among 1,920 (20.7\%), and information regarding age at death was missing among 887 (9.6\%, Table 2). In the MADT study, the twins in 1,978 (99.2\%), 1,581 (99.2\%), and 1,306 (98.9\%) intact pairs reported their father's, mother's, or both parents' age at death within \pm 4 years. In the LSADT, the twins in 473 (88.6\%), 490 (89.4\%), and $428(80.8 \%)$ intact pairs reported their father's, mother's, and both parents' age at death within \pm 4 years.

Mean parental age at death was only modestly associated with physical and cognitive functioning, as seen in Figures 1-3 and Table 3. For each 10-year increase in parental life span, average grip strength increased by only $0.32 \mathrm{~kg}(95 \% \mathrm{CI}=0.00-0.63)$ (Table 3$)$. The effect was seen only in the LSADT cohort (mean age mid-70s), but not among the twins who were middleaged (MADT), or the cohort of nonagenarians (1905). Likewise, the relation of cognitive functioning to parental age at death was seen solely in the LSADT cohort; each 10-year increase in parental age at death was associated with an increase of $0.20(95 \% \mathrm{CI}=0.03-0.37)$ MMSE points and $0.24(95 \% \mathrm{CI}=0.07-0.40)$ cognitive composite points (Table 3). The analyses used for Table 3 were repeated using parental age at death as a continuous variable in the multivariate linear regression models. The coefficients were, when multiplied by 10 , virtually identical to the ones in Table 3 (results not shown).

A number of self-reported diseases were associated with parental life span (Table 4). Again the effect was mainly present in the LSADT cohort. In this cohort the age- and sex-adjusted odds ratios (ORs) for having either previous or current diabetes, hypertension, ischemic theart disease, heart failure, or stroke each decreased by roughly 0.20 for every 10 -year increase in mean parental age at death (Table 4). Also, the odds for having fair, poor, or very poor self-rated health were associated with parental age at death: $\mathrm{OR}=0.8(0.7$ to 0.9$)$ in both the MADT and LSADT cohorts by 10 -year increase in parental age at death. Pulmonary disease, cancer, osteoarthritis, and osteoporosis were not associated with pa-

TABLE 2. Number of Participants (Percentages) in Three Large Danish Population-Based Studies Stratified by Mean Parental Age at Death

\begin{tabular}{|c|c|c|c|c|c|c|c|c|c|c|c|c|c|c|c|c|c|c|}
\hline & \multicolumn{10}{|c|}{ Mean Parental Age at Death (Both Parents Dead) } & \multirow{2}{*}{\multicolumn{4}{|c|}{$\begin{array}{c}\text { Two } \\
\text { Parents } \\
\text { Alive }\end{array}$}} & \multirow{2}{*}{\multicolumn{2}{|c|}{ Unknown }} & \multirow{2}{*}{\multicolumn{2}{|c|}{ Total }} \\
\hline & \multicolumn{2}{|c|}{$<54$} & \multicolumn{2}{|c|}{$55-64$} & \multicolumn{2}{|c|}{$65-74$} & \multicolumn{2}{|c|}{$75-84$} & \multicolumn{2}{|c|}{$85+$} & & & & & & & & \\
\hline & No. & $\%$ & No. & $\%$ & No. & $\%$ & No. & $\%$ & No. & $\%$ & No. & $\%$ & No. & $\%$ & No. & $\%$ & No. & $\%$ \\
\hline MADT & 82 & 1.9 & 257 & 6.0 & 680 & 15.8 & 889 & 20.6 & 256 & 5.9 & 1,389 & 32.2 & 516 & 12.0 & 245 & 5.7 & 4,314 & 100 \\
\hline 1905 & 89 & 3.9 & 240 & 10.6 & 514 & 22.7 & 666 & 29.4 & 326 & 14.4 & 0 & 0.0 & 0 & 0.0 & 427 & 18.9 & 2,262 & 100 \\
\hline
\end{tabular}

MADT $=$ Study of Middle-Aged Danish Twins; LSADT = Longitudinal Study of Aging Danish Twins—3rd wave; $1905=$ Danish 1905 Cohort Study. 


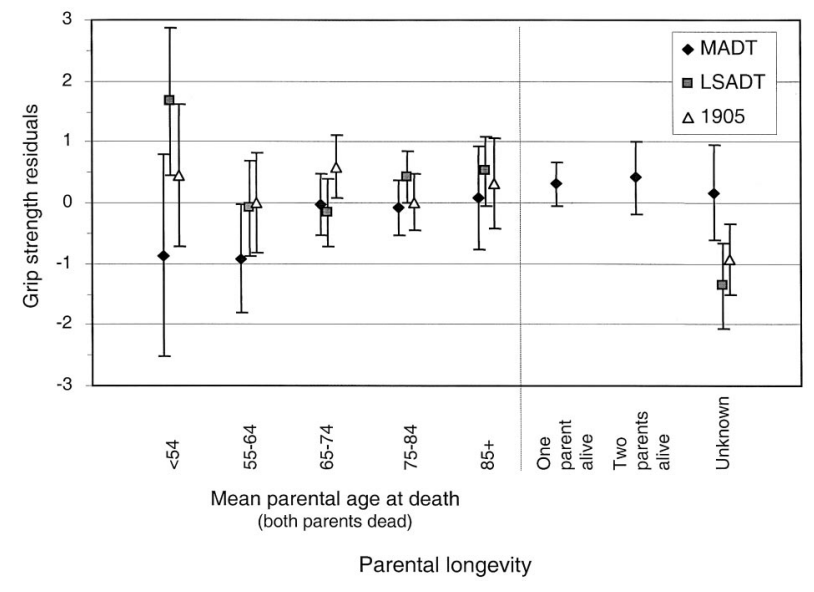

FIGURE 1. Grip strength by parental longevity (age- and sex-adjusted residuals) among 9,285 participants in three surveys: the Study of Middle-Aged Danish Twins (MADT), the Longitudinal Study of Aging Danish Twins-3rd wave (LSADT), and the Danish 1905 Cohort Survey (1905).

rental life span in any cohort (Table 4). In the 1905 cohort, parental life span was not associated with any disease or self-rated health. In the MADT cohort there was a borderline association of parental life span with hypertension and stroke, but not with any other disease (Table 4). Because the associations observed between parental life span and stroke, ischemic heart disease, and heart failure could reflect confounding from the association also seen with hypertension and diabetes, we subsequently used a model for/stroke, ischemic heart disease, and heart failure including hypertension and diabetes only in the LSADT sample. The point esti-

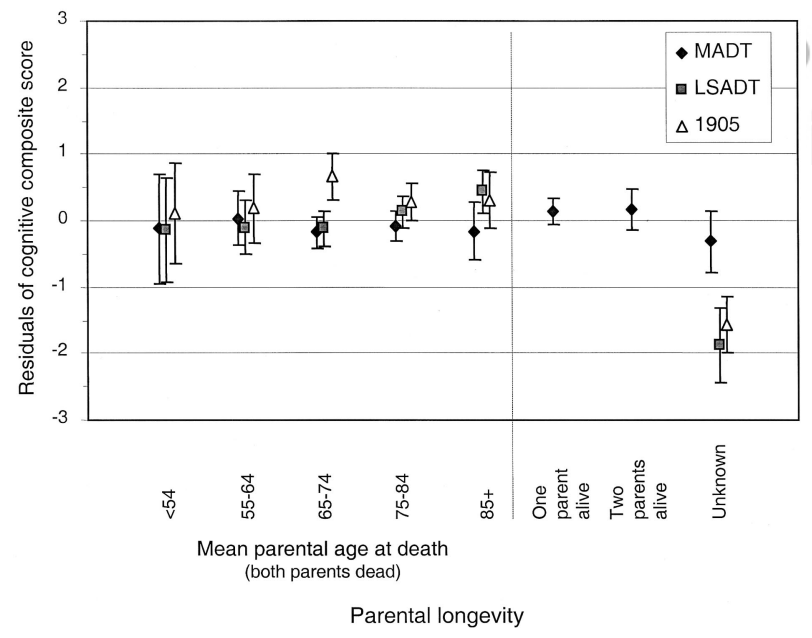

FIGURE 2. Cognitive composite score by parental longevity (age- and sex-adjusted residuals) among 9,285 participants in three surveys: the Study of Middle-Aged Danish Twins (MADT), the Longitudinal Study of Aging Danish Twins3rd wave (LSADT), and the Danish 1905 Cohort Survey (1905).

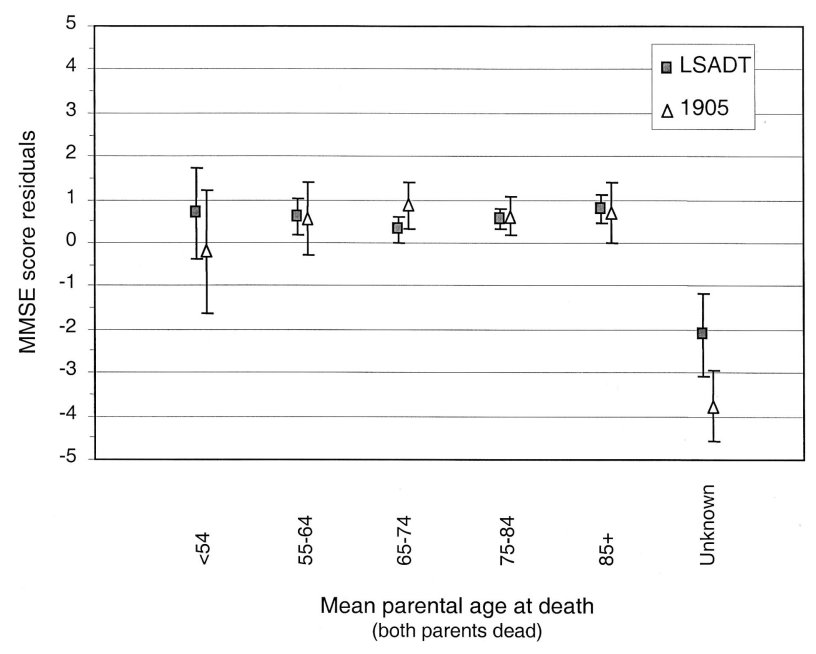

FIGURE 3. MMSE score by parental longevity (age- and sex-adjusted residuals) among participants in two surveys: the Longitudinal Study of Aging Danish Twins-3rd wave (LSADT), and the Danish 1905 Cohort Survey (1905).

mates from these models for these three diseases were virtually unaltered.

\section{Discussion}

The relationship between long-lived parents and successful aging has been reported in one previous paper. ${ }^{20}$ Vaillant studied a cohort of 188 males age 65 years who had been recruited in 1940-1942 as healthy students from the top half of their classes in college (Harvard University). When the $16 \%$ with longest maximum ancestral longevity were compared with the $16 \%$ with shortest ancestral longevity, long-lived ancestors predicted "sustained health" on a crude 1-5 scale.

A number of issues in our study need to be addressed. The information about parental life span was based on aself-report from the participants, which could be subject to error, although it is reassuring that the twins within the intact pairs reported their parents' age at death with high correlations. The proportion able to report the parental age at death $( \pm 3$ years $)$ compared with death certificates has previously been found in Sweden to be between $82.8 \%$ and $86.5 \%$ for fathers and $86.7 \%$ and $90.2 \%$ for mothers. ${ }^{21}$

To estimate the effect of potential misclassification of parental age at death, we repeated the analyses excluding those in which the interviewers stated that the interview was completed with difficulties (of any kind and severity) $(\mathrm{N}=1,324)$. The point estimates after this restriction were very similar to those reported here, although the confidence intervals widened.

We report here the results of the analyses using the average of the mothers' and fathers' age at death as the covariate of interest. This approach enabled us to use the 
TABLE 3. Physical and Cognitive Functioning Among 9,285 Participants in Three Large Danish Population-Based Studies in Relation to Parental Age at Death

\begin{tabular}{|c|c|c|c|c|c|c|}
\hline & \multicolumn{6}{|c|}{ Average Change by 10 -Year increase in Average Parental Age at Death } \\
\hline & \multicolumn{2}{|c|}{ MADT } & \multicolumn{2}{|c|}{ LSADT } & \multicolumn{2}{|c|}{1905} \\
\hline & Average & $95 \% \mathrm{CL}$ & Average & $95 \% \mathrm{CL}$ & Average & $95 \% \mathrm{CL}$ \\
\hline $\begin{array}{l}\text { Physical function } \\
\text { Maximum hand-grip strength, kg* } \\
\text { Cognitive function }\end{array}$ & 0.14 & $-0.27,0.55$ & 0.32 & $0.00,0.63$ & -0.02 & $-0.35,0.30$ \\
\hline $\begin{array}{l}\text { MMSE score } \dagger \\
\text { Cognitive composite score }\end{array}$ & -0.06 & $-0.25,0.13$ & $\begin{array}{l}0.20 \\
0.24\end{array}$ & $\begin{array}{l}0.03,0.37 \\
0.07,0.40\end{array}$ & $\begin{array}{r}0.00 \\
-0.05\end{array}$ & $\begin{array}{l}-0.31,0.32 \\
-0.24,0.15\end{array}$ \\
\hline
\end{tabular}

The numbers are average change in the respective measure by 10 -year increase in mean parental age at death with $95 \%$ confidence intervals in brackets. The coefficients are adjusted for age and sex. MADT = Study of Middle-Aged Danish Twins; LSADT = Longitudinal Study of Aging Danish Twins-3rd wave; $1905=$ Danish 1905 Cohort Study; MMSE = Mini Mental State Examination.

* Maximum of at least three examinations with less than $20 \mathrm{~kg}$ difference between two measures.

$\uparrow$ Only cohorts from the Danish 1905 Cohort Study and the Longitudinal Study of Aging Danish Twins.

\$ A composite measure of test for fluency, forward and backward digit span, and a modified 12-word learning test.

information from those with two long-lived parents, a group that could be expected to experience "successful aging." To get an impression of parental-specific effects, we also analyzed the effects of fathers' and mothers' ages at death separately. However, the results were not systematically different between the two (results not shown). The results from the participants with one or two parents still alive were censored from the analyses. As seen in Table 2, the number of participants omitted for this reason was only considerable in the MADT cohort and may have contributed to the lāck of assōciation between parental age at death and the outcomes in this cohort.

Participants who did not provide information on parental age at death were more/cognitively impaired than the rest (Figures 2-3). These participants were also approximately 10 years older (mean $81.2 \pm 14.2$ ) than participants without missing information (mean $70.9 \pm$ 15.8). The direction of the potential bias introduced by the lack of information from these participants is not evident.
When analyzing MMSE score as the outcome, we used methods that rely on a normal distribution of the data. Because the MMSE score is highly skewed, we repeated the regressions using bootstrap methods. ${ }^{22}$ The confidence intervals differed only in the third decimal place (data not shown).

We found that parental life span is associated with physical and cognitive functioning and health in the cohort of $70+$ year olds. Age-related differences in functioning and health among middle-aged individuals may not be large enough to detect a small effect of parental longevity. In the nonagenarian 1905 cohort, it would have beên surprising if parental life span had had an impact, because reaching an age of $92+$ may in itself be regarded as successful aging.

In conclusion, we found that at older ages parental life span is positively associated with physical and cognitive functioning and avoidance of some of the quantitatively important diseases. However, the overall effects are small in absolute terms and are seen only among

TABLE 4. Self-Reported* Diseases and Health Among 9,285 Participants in Three Large Danish Population-Based Studies in Relation to Parental Age at Death

\begin{tabular}{|c|c|c|c|c|c|c|}
\hline & \multicolumn{6}{|c|}{ Odds Ratio for Each 10-Year Increase in Average Parental Age at Death } \\
\hline & \multicolumn{2}{|c|}{ MADT } & \multicolumn{2}{|c|}{ LSADT } & \multicolumn{2}{|c|}{1905} \\
\hline & OR & $95 \% \mathrm{CI}$ & OR & $95 \% \mathrm{CI}$ & OR & $95 \% \mathrm{CI}$ \\
\hline Diabetes & 0.86 & $0.64-1.14$ & 0.82 & $0.69-0.96$ & 1.04 & $0.85-1.27$ \\
\hline Pulmonary disease $\dagger$ & 0.92 & $0.78-1.09$ & 0.96 & $0.85-1.09$ & 0.93 & $0.80-1.08$ \\
\hline Hypertension & 0.88 & $0.77-1.00$ & 0.86 & $0.78-0.96$ & 0.92 & $0.81-1.03$ \\
\hline Ischemic heart disease $\$$ & 0.88 & $0.72-1.07$ & 0.82 & $0.73-0.94$ & 0.90 & $0.78-1.03$ \\
\hline Heart failure & 0.90 & $0.72-1.12$ & 0.81 & $0.70-0.93$ & 0.96 & $0.85-1.10$ \\
\hline Stroke & 0.71 & $0.50-1.00$ & 0.76 & $0.64-0.90$ & 0.90 & $0.76-1.07$ \\
\hline Cancer excluding skin & 1.03 & $0.75-1.40$ & 1.00 & $0.84-1.41$ & 1.04 & $0.87-1.25$ \\
\hline Osteoarthritis & 0.90 & $0.79-1.03$ & 1.05 & $0.95-1.16$ & 1.02 & $0.92-1.14$ \\
\hline Osteoporosis & 0.97 & $0.69-1.36$ & 0.99 & $0.82-1.20$ & 0.98 & $0.83-1.16$ \\
\hline Fair, poor, or very poor self-rated health & 0.78 & $0.69-0.89$ & 0.84 & $0.76-0.92$ & 0.93 & $0.84-1.04$ \\
\hline
\end{tabular}

Values are age- and sex-adjusted odds ratios and $95 \%$ confidence intervals in the respective measure by 10 -year increase in mean parental age at death.

* Participants were asked if a physician ever had told them that they suffered from any of the mentioned diseases

MADT = Study of Middle-Aged Danish Twins; LSADT = Longitudinal Study of Aging Danish Twins-3rd wave; $1905=$ Danish 1905 Cohort Study.

$\dagger$ Asthma and/or chronic bronchitis.

\$ Angina and/or acute myocardial infarction. 
the elderly (mean age mid-70s), but not among the middle-aged or the oldest old.

\section{References}

1. Rowe JW, Kahn RL. Human aging: usual and successful. Science 1987;237:143-149.

2. Guralnik JM, Kaplan GA. Predictors of healthy aging: prospective evidence from the Alameda County study. Am J Public Health 1989;79:703-708.

3. Strawbridge WJ, Cohen RD, Shema SJ, Kaplan GA. Successful aging: predictors and associated activities. Am J Epidemiol 1996; 144:135-141.

4. Jonker C, Schmand B, Lindeboom J, Havekes LM, Launer LJ. Association between apolipoprotein E epsilon4 and the rate of cognitive decline in community-dwelling elderly individuals with and without dementia. Arch Neurol 1998;55:1065-1069.

5. Corder EH, Saunders AM, Strittmatter WJ, et al. Gene dose of apolipoprotein E type 4 allele and the risk of Alzheimer's disease in late onset families. Science 1993;261:921-923.

6. Wilson PW, Schaefer EJ, Larson MG, Ordovas JM. Apolipoprotein $\mathrm{E}$ alleles and risk of coronary disease: a meta-analysis. Arterioscler Thromb Vasc Biol 1996;16:1250-1255.

7. Christensen K, McGue M, Yashin A, Iachine I, Holm NV, Vaupel JW. Genetic and environmental influences on functional abilities in Danish twins aged 75 years and older. J Gerontol A Biol Sci Med Sci 2000;55:M446-M452.

8. McClearn GE, Johansson B, Berg S, et al. Substantial genetic influence on cognitive abilities in twins 80 or more years old. Science 1997;276:1560-1563.

9. McGue M, Christensen K. The heritability of cognitive functioning in the very old: evidence from Danish twins aged 75 and older. Psychol Aging 2001;16:272-280.

10. Herskind AM, McGue M, Holm N , Sørensen TIA, Harvald B, Vaupel JW. The heritability of human longevity:-a populationbased study of 2872 Danish twin pairs born 1870-1900. Hum Genet 1996;97:319-323.
11. Marenberg ME, Risch N, Berkman LF, Floderus B, de Faire U. Genetic susceptibility to death from coronary heart disease in a study of twins. N Engl J Med 1994;330:1041-1046.

12. Poulsen P, Kyvik KO, Vaag A, Beck-Nielsen H. Heritability of type II (non-insulin-dependent) diabetes mellitus and abnormal glucose tolerance: a population-based twin study. Diabetologia 1999;42:139-145.

13. Lichtenstein P, Holm NV, Verkasalo PK, et al. Environmental and heritable factors in the causation of cancer: analyses of cohorts of twins from Sweden, Denmark, and Finland. N Engl J Med 2000; 343:78-85.

14. Gaist D, Bathum L, Skytthe A, et al. Strength and anthropometric measures in identical and fraternal twins: no evidence of masculinization of females with male co-twins. Epidemiology 2000;11: 340-343.

15. Christensen K, Holm NV, McGue M, Corder L, Vaupel JW. A Danish population-based twin study on general health in the elderly. J Aging Health 1999;11:49-64.

16. Nybo H, Gaist D, Jeune B, et al. The Danish 1905 cohort: a genetic-epidemiological nationwide survey. J Aging Health 2001; 13:32-46.

17. Kyvik KO, Christensen K, Skytthe A, Harvald B, Holm NV. The Danish Twin Register. Dan Med Bull 1996;43:467-470.

18. Rantanen T, Pertti E, Kauppinen M, Heikkinen E. Maximal isometric muscle strength and socioeconomic status, health, and physical activity in 75-year-old persons. J Aging Phys Activity 1994:2:206-220.

19. McGue M, Bouchard TJ Jr. Adjustment of twin data for the effects of age and sex. Behav Genet 1984;14:325-343.

20. Vaillant GE. The association of ancestral longevity with successful aging. J Gerontol 1991;46:P292-P298.

21. Hedstrand $\mathrm{H}$, Waern $\mathrm{U}$, Aberg $\mathrm{H}$. The reliability of information obtained from middle-aged men about the causes of their parents' death and their age at death (in Swedish). Lakartidningen 1976; 73:503-504.

22. Efron B, Tibshirani R. Bootstrap methods for standard errors, confidence intervals, and other measures of statistical accuracy. Stat Sci 1986;1:54-77.

\section{Unauthorized Use Prohibited}

